\title{
Tumor-induced acute pancreatitis
}

\begin{abstract}
Alcohol and biliary stones are the predominant etiological factors for acute pancreatitis. Commonly, acute pancreatitis is associated with a decrease in serum calcium levels. Hypercalcemia as a cause of pancreatitis has been rarely reported. In the presence of hypercalcemia, primary hyperparathyroidism due to benign parathyroid adenoma is the most common etiology. Surgical resection of parathyroid adenoma is the definitive management with excellent results. Thus, it is prudent to monitor serum calcium levels in patients with acute pancreatitis.
\end{abstract}

Keywords: pancreatitis, hypercalcemia, hyperparathyroidism
Volume 8 Issue $6-2018$

\author{
Mandeep Singla,' Abhinav Gupta ${ }^{2}$ \\ 'Department of General Medicine, Govt. Medical College and \\ Hospital, India \\ ${ }^{2}$ Department of General Medicine, Govt. Medical College and \\ Hospital, India
}

Correspondence: Mandeep Singla, Department of General Medicine, Govt. Medical College and Hospital, India Email drmamdeep2007@gmail.com

Received: May 20, 2018 | Published: December 04, 2018

\section{Introduction}

Gall stones and alcohol are the predominant etiological factors for acute pancreatitis. Commonly, acute pancreatitis is associated with a decrease in serum calcium levels. Hypercalcemia as a cause of pancreatitis has been rarely reported. Herein, we report a young female who had hypercalcemia-related acute pancreatitis as a presenting manifestation of solitary parathyroid adenoma.

\section{Case report}

A 30-year-old female presented to the emergency department with severe epigastric pain and persistent vomiting for two days. Physical examination revealed tenderness and guarding in the epigastric area. Bowel sounds were sluggish. Laboratory investigations revealed raised serum amylase (910U/1, reference range 28-100U/1) and serum lipase (286U/1, reference range $<67 \mathrm{U} / 1$ ) levels. Computed tomography of the abdomen revealed bulky pancreas with peripancreatic fat stranding, thus confirming acute pancreatitis. Further biochemical investigations revealed high serum calcium $(3.5 \mathrm{mmol} / \mathrm{L})$ with low serum phosphate $(0.56 \mathrm{mmol} / \mathrm{L})$. Serum parathyroid hormone levels were markedly elevated $(638 \mathrm{ng} / \mathrm{L})$ with normal $25(\mathrm{OH})$ vitamin D levels $(84 \mathrm{nmol} / \mathrm{L})$. Computed tomography of the neck revealed $5 \times 3 \mathrm{~cm}$ heterogeneously enhancing soft tissue lesion in the left retrotracheal space displacing the left lobe of thyroid gland anteriorly (Figure 1A). Technetium (Tc-99m) labeled sestamibi scan revealed the presence of hot focus in the left inferior pole of thyroid in the delayed image, consistent with parathyroid adenoma (Figure 1B). The patient received fluid replacement with saline and bisphosphonates. After therapy, the serum calcium levels decreased, and the acute pancreatitis subsided. Patient underwent surgical excision of adenoma, and histopathology confirmed the diagnosis.

\section{Discussion}

Hyperparathyroidism -induced hypercalcemia is a rare cause of acute pancreatitis. It was first described in $1957 .{ }^{1}$ The prevalence of acute pancreatitis in primary hyperparathyroidism has been estimated to be between 1.5 and $13 \%$. The pathophysiology of acute pancreatitis induced by hypercalcemia remains unknown. The postulated mechanism is accelerated intrapancreatic conversion of trypsinogen to trypsin by hypercalcemia, leading to autodigestion of the pancreas and pancreatic injury. ${ }^{2}$ Further, sustained hypercalcemia can lead to pancreatic duct obstruction by the deposition of calcium in the pancreatic duct. ${ }^{3}$ Usually, acute pancreatitis is associated with a decrease in serum calcium and constitutes a prognosis factor during the first 48hours of admission. ${ }^{4}$ Therefore, the presence of hypercalcemia increases the diagnostic suspicion of hyperparathyroidism. Vigorous fluid resuscitation with normal saline and bisphosphonate therapy are the mainstay of management in hypercalcemia. The preoperative localization of parathyroid adenoma is performed by means of a neck ultrasound, neck tomography or scintigram with sestamibi. Surgical excision of the parathyroid adenoma is the definite treatment with $100 \%$ cure rate and prevents recurrence of acute pancreatitis.

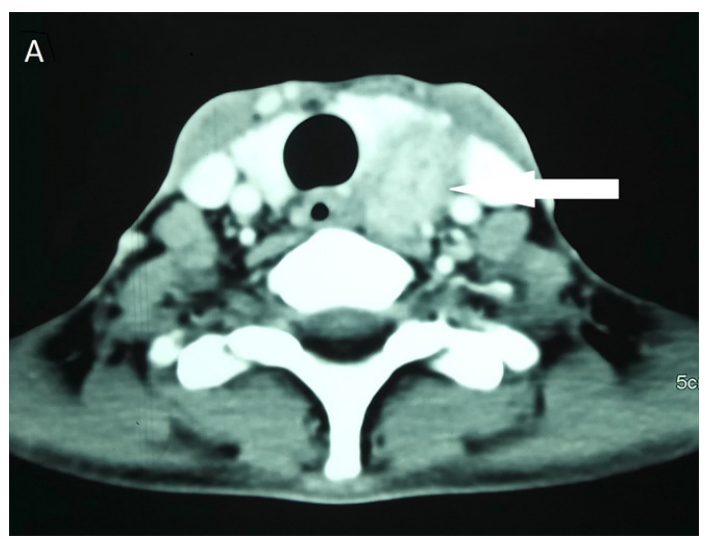

Figure I Computed tomography of the neck revealed $5 \times 3 \mathrm{~cm}$ heterogeneously enhancing soft tissue lesion in the left retotracheal space

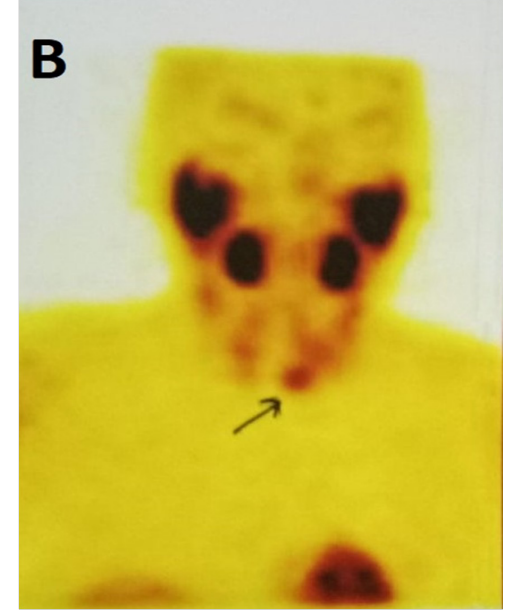

Figure 2 Technetium (Tc-99m) labeled sestamibi scan revealed the presence of hot focus in the left inferior pole of thyroid in the delayed image, consistent with parathyroid adenoma. 


\section{Conclusion}

Commonly, there is a fall in serum calcium levels in patients with acute pancreatitis. However, hyperparathyroidism-induced hypercalcemia per se can lead to development of acute pancreatitis. These patients have high serum calcium levels in the setting of acute pancreatitis. The definitive management includes surgical resection of parathyroid adenoma yielding excellent results. Thus, it is prudent to monitor serum calcium levels in patients with acute pancreatitis.

\section{Acknowledgments}

None.

\section{Conflicts of interest}

The authors have no conflicts of interest to declare

\section{References}

1. Cope O, Culver PJ, Mixter CG Jr, et al. Pancreatitis, a diagnostic clue to hyperparathyroidism. Ann Surg. 1957;145(6):857-863.

2. Mithöfer K, Fernández-del Castillo C, Frick TW, et al. Acute hypercalcemia causes acute pancreatitis and ectopic trypsinogen activation in the rat. Gastroenterology. 1995;109(1):239-246.

3. Ward JB, Petersen OH, Jenkins SA, Sutton R. Is an elevated concentration of acinar cytosolic free ionised calcium the trigger for acute pancreatitis? Lancet. 1995;346(8981):1016-1019.

4. Ranson JH. Etiological and prognostic factors in human acute pancreatitis: a review. Am J Gastroenterol. 1982;77(9):633-638. 